\title{
Modelo para Pré-Dimensionamento de Bacias de Detenção para Controle da Poluição Difusa das Águas Pluviais no Município de Porto Alegre
}

\author{
Marcelo Kipper da Silva e André Luiz Lopes da Silveira \\ Instituto de Pesquisas Hidráulicas - UFRGS \\ mk.silva@terra.com.br,andre@iph.ufrgs.br
}

Recebido: 08/09/10 - revisado: 17/01/11 - aceito: 04/11/11

\begin{abstract}
RESUMO
A poluição de origem difusa das águas pluviais é um entre os vários impactos causados pela urbanização. As bacias de detenção são usadas para controlar as vazões de pico, mas também podem ser usadas para o controle da qualidade da água através da sedimentação natural dos poluentes. Neste trabalho foi desenvolvido um modelo de pré-dimensionamento de bacias de detenção para o controle da poluição difusa no Município de Porto Alegre, baseado em uma metodologia de maximização de captura de volume e em uma metodologia de cálculo da eficiência da remoção de poluentes. No desenvolvimento do modelo foram utilizadas séries históricas de precipitações ocorridas em Porto Alegre. Os resultados das simulações mostraram que o volume de detenção necessário para o controle da poluição difusa é de $157 \mathrm{~m}^{3} / \mathrm{ha}$ para um coeficiente de escoamento de 0,6, típico de áreas urbanas, variando de 36 a $225 \mathrm{~m}^{3} / \mathrm{ha}$ para coeficientes de escoamento de 0,1 e 1,0, respectivamente. $O$ modelo proposto foi comparado com modelos aplicados em locais nos Estados Unidos com características de precipitação semelhantes, resultando em volumes de detenção compatíveis.
\end{abstract}

Palavras-chave: bacia de detenção; poluição difusa, águas pluviais.

\section{INTRODUÇÃO}

A urbanização não planejada gera sérios impactos sobre a drenagem de uma cidade. Ela faz com que sejam impermeabilizadas grandes porções do solo, promovendo aumento da vazão de pico dos hidrogramas e a antecipação, no tempo, desse pico.

Para controlar os efeitos da urbanização sobre o aumento do escoamento, em muitos locais nos EUA, foram construídas bacias de detenção (Chaney, 1982). Estas obras tinham como enfoque principal a redução dos custos totais, pois em muitos casos, era mais barato construir tais bacias do que ampliar a capacidade de escoamento das redes existentes.

Juntamente com os problemas decorrentes da alteração das características hidrológicas das bacias hidrográficas causadas pela urbanização, existem problemas relacionados à qualidade das águas urbanas. Pesquisas mostraram que parte da poluição gerada em áreas urbanas se origina do escoamento superficial sobre áreas impermeáveis, áreas em fase de construção, depósitos de lixo ou de resíduos industriais, etc (Porto, 1995), sendo denominada de poluição difusa.
A partir do surgimento da preocupação com a poluição difusa em meados dos anos 80 , o controle da qualidade das águas pluviais passou a fazer parte dos critérios adotados no projeto e gerenciamento das obras. Atualmente as autoridades responsáveis pelo gerenciamento e aprovação dos projetos de urbanização em alguns países desenvolvidos exigem um controle integrado tanto dos aspectos quantitativos quanto qualitativos (Amec, 2001; Maryland, 2000).

Segundo Clar e Barfield (2004) os projetos visando o controle qualitativo das águas estão focados no volume anual de escoamento superficial ao invés de vazões de pico de eventos. Isto ocorre porque todos os eventos de precipitação e o seu conseqüente escoamento superficial carregam poluentes. Apesar da concentração de poluentes ser maior no início do escoamento em alguns eventos, devido à ocorrência de carga de lavagem, o restante do escoamento também contém poluentes e, portanto, deve ser tratado.

Estudos mostram que as chuvas menos intensas, porém com períodos de retorno que vão de dias até um ano respondem pela maior parte do volume de escoamento anual. Analisando a variabilidade das chuvas em Denver, Guo e Urbonas (1996) concluíram que $94,1 \%$ do volume anual de precipi- 
tação é causado por chuvas com menos de um ano de período de retorno. Comportamentos semelhantes são observados em outros locais (Casqa, 2002). Portanto, quando se abordam os aspectos qualitativos das águas pluviais, é necessário levar em conta todos os eventos de chuva que produzem escoamento.

Para que ocorra a remoção dos poluentes através da sedimentação, é necessário que a água permaneça dentro da bacia de detenção o maior tempo possível, pois existem sólidos suspensos de pequenas dimensões que têm uma velocidade de sedimentação muito pequena. Devido às limitações de ordem prática, o tempo de esvaziamento da água na bacia de detenção deve ser limitado. Valores típicos são de 12, 24 ou 48 horas.

Como todos os eventos de escoamento gerados pela precipitação carregam poluentes, em uma situação ideal, todo o escoamento deveria ser tratado. Como isto não é possível, a questão a ser respondida é qual o volume de escoamento superficial que deve ser capturado e tratado por uma estrutura? Sabe-se que este é um fator crítico para o sucesso do tratamento. Se o volume do reservatório for muito pequeno, muitos eventos irão exceder a capacidade da estrutura e grande parte do escoamento irá sair da estrutura carregando poluentes, ou seja, a eficiência do tratamento será pequena. Por outro lado, se o volume da bacia de detenção for muito grande, o custo da estrutura será excessivamente alto.

Em suma, duas variáveis conflitantes são necessárias para o dimensionamento de uma estrutura de controle qualitativo: o tempo de esvaziamento da bacia e o volume de armazenamento da bacia. $O$ tempo de esvaziamento é estabelecido no início da análise para dimensionamento. $\mathrm{O}$ volume depende das características da precipitação local que está sendo considerada tais como: volume do evento, duração do evento e intervalo de tempo entre eventos consecutivos. Se a precipitação ao longo do ano consiste de poucos eventos com grande volume, a bacia de detenção terá um volume maior do que se ocorressem muitos eventos de pequeno volume. Se os eventos forem de curta duração, a bacia terá um volume maior do que se os eventos tiverem uma grande duração. Se o intervalo de tempo for muito curto entre eventos o volume será maior, pois a bacia pode não estar totalmente vazia quando o evento seguinte iniciar.

O objetivo deste trabalho é propor um modelo de pré-dimensionamento de bacias de detenção para o controle da poluição difusa no Município de Porto Alegre, combinando um método de maxi- mização de captura de volume com um método de análise de eficiência da remoção de sólidos em suspensão.

Para o desenvolvimento do modelo foi utilizada uma série de dados de precipitação do posto pluviográfico do Oitavo Distrito de Meteorologia do INMET.

\section{CÁLCULO DO VOLUME MAXIMIZADO DE CONTROLE}

O método de Urbonas e Starhe (1993) visa a otimização do volume de escoamento capturado e tratado. A Figura 1 auxilia a compreensão da metodologia. O eixo horizontal representa o volume normalizado da bacia de detenção, enquanto que o eixo vertical representa a fração do volume total capturado. Partindo-se de um ponto próximo a intersecção dos eixos, um pequeno incremento no volume da bacia de detenção produz um grande aumento no volume capturado. A medida que aumenta o volume de detenção, um incremento neste volume produz um aumento cada vez menor no volume capturado até ser atingido o ponto de maximização. No ponto de maximização a declividade da reta tangente é de 1:1, ou seja, a razão entre os volumes é igual a 1. A partir do ponto de maximização a razão entre os aumentos de volumes se inverte, ou seja, é necessário um aumento cada vez maior no volume da bacia de detenção para se obter um determinado aumento no volume capturado.

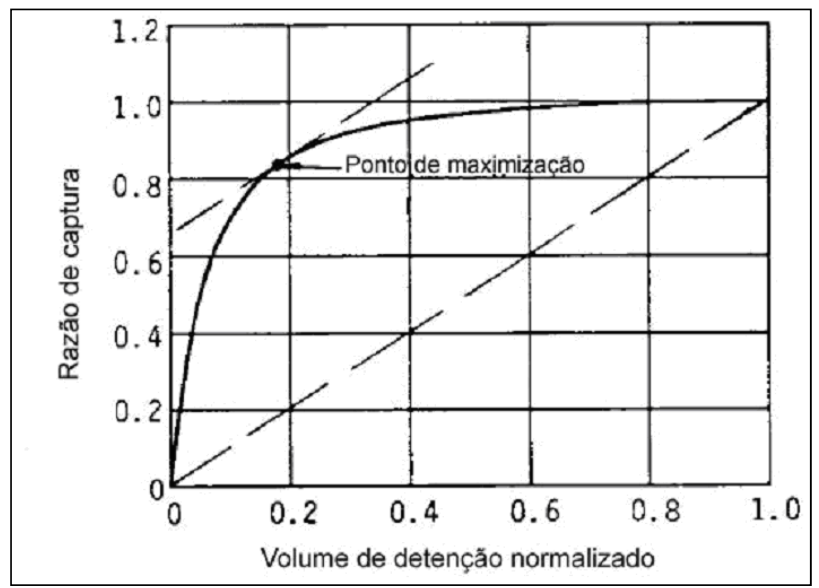

Figura 1 - Maximização do volume de controle (adaptado de Guo e Urbonas, 1996). 
Para um evento qualquer o volume de precipitação pode ser convertido em escoamento através da Equação (1):

$\mathrm{P}_{\mathrm{r}}=\mathrm{C}^{*}\left(\mathrm{P}_{\mathrm{t}}-\mathrm{P}_{\mathrm{i}}\right)$

onde: $\mathrm{P}_{\mathrm{r}}=$ volume de escoamento $(\mathrm{mm})$;

$\mathrm{C}=$ coeficiente de escoamento;

$(\mathrm{mm})$;

$\mathrm{P}_{\mathrm{t}}=$ precipitação sobre a área contribuinte

$\mathrm{P}_{\mathrm{i}}=$ perdas iniciais $(\mathrm{mm})$.

Para uma bacia de detenção com um volume total dado por $\mathrm{P}_{\mathrm{P}}(\mathrm{mm})$, a vazão média de esvaziamento $\mathrm{q}_{\mathrm{s}}(\mathrm{mm} / \mathrm{h})$ é dada por:

$\mathrm{q}_{\mathrm{s}}=\mathrm{P}_{\mathrm{P}} / \mathrm{T}_{\mathrm{e}}$

onde: $\mathrm{T}_{\mathrm{e}}=$ tempo de esvaziamento do volume total da bacia em horas;

$\mathrm{P}_{\mathrm{P}}=$ volume da bacia de detenção em $\mathrm{mm}$ por unidade de área contribuinte.

O volume máximo de escoamento que a bacia pode capturar é igual ao seu volume mais o volume de água que é liberado pela estrutura de saída durante a duração do evento:

$\mathrm{P}_{\mathrm{C}}=\mathrm{P}_{\mathrm{P}}+\mathrm{q}_{\mathrm{s}} * \mathrm{~T}_{\mathrm{d}}$

onde: $\quad \mathrm{P}_{\mathrm{c}}=$ máximo volume capturado $(\mathrm{mm})$;

$\mathrm{T}_{\mathrm{d}}=$ duração do evento $(\mathrm{h})$.

Se o volume de escoamento for menor que o volume máximo de captura, considera-se que o evento é inteiramente capturado e tratado. Se o volume de escoamento for maior que o volume máximo de captura, o volume em excesso é considerado como volume não tratado.

Para um conjunto de $\mathrm{N}$ eventos que produzem escoamento, somando-se o volume de escoamento de todos estes, tem-se o volume acumulado para o período, dado por $\mathrm{P}_{\mathrm{tr}}$, em $\mathrm{mm}$ :

$P_{t r}=\sum_{j=1}^{N} \operatorname{Pr}_{j}$

$\mathrm{O}$ volume acumulado não capturado $\mathrm{P}_{\text {to }}$, é dado por:

$P_{t o}=\sum_{j=1}^{N}\left(\operatorname{Pr}_{r}-P_{c j}\right)$
A partir destes dois volumes dados pelas Equações (4) e (5) pode-se definir uma razão de captura de escoamento $\mathrm{R}_{\mathrm{v}}$ para o todo o período considerado:

$\mathrm{R}_{\mathrm{v}}=1-\left(\mathrm{P}_{\mathrm{to}} / \mathrm{P}_{\mathrm{tr}}\right)$

Antes da aplicação deste método, é necessária a escolha do tempo de esvaziamento da bacia, que deve ser igual ao dobro do período de tempo sem precipitação usado para delimitação de eventos de precipitação. Os eventos de precipitação são convertidos em escoamento através da adoção de um coeficiente de escoamento e da aplicação da Equação (1). Em seguida, são ordenados em ordem crescente de volumes escoados superficialmente. Para evitar que poucos eventos de grande magnitude influenciem os resultados, o valor que corresponde ao percentil 99,5 $\left(\mathrm{P}_{\mathrm{M}}\right)$ foi selecionado e usado como o maior evento na análise. Este valor também é usado para normalizar o volume de detenção da bacia.

Para encontrar o valor otimizado de controle, foi testada uma série de valores incrementais de volumes de detenção. Para cada um dos valores da série, calculou-se a razão de captura de escoamento através das Equações (4), (5) e (6). Em seguida, foi confrontado o valor da razão de captura de escoamento (eixo vertical) contra o volume de detenção, normalizado pelo volume correspondente ao percentil 99,5. O ponto de maximização foi determinado através da procura do ponto cuja declividade da reta tangente é igual a 1.

\section{ANÁLISE DA EFICIÊNCIA DA REMOÇÃO DE POLUENTES}

Para a avaliação da eficiência na remoção de sólidos em suspensão em bacias de detenção, Driscoll et al. (1986) apresenta um método que combina modelos estatísticos de análise de precipitação e escoamento superficial com um modelo de sedimentação para a remoção de sólidos em suspensão sob condições estáticas e dinâmicas. A condição dinâmica ocorre durante os eventos de precipitação, quando há vazão afluente à bacia de detenção. Por outro lado a condição estática ocorre quando não há precipitação, mas ainda existe um volume de água na bacia, permitindo a sedimentação dos sólidos em suspensão e poluentes. 
Para a utilização deste método é necessário o conhecimento dos valores médios e coeficientes de variação de quatro variáveis relacionadas com o escoamento superficial que ocorre em um determinado local de interesse: volume, vazão, duração e intervalo de tempo entre eventos consecutivos.

Também é necessário que se conheça a distribuição granulométrica dos sólidos em suspensão contidos na água afluente à bacia de detenção, pois a velocidade de sedimentação depende diretamente do tamanho das partículas em suspensão. Por este motivo, o método permite o cálculo da eficiência da sedimentação por faixas de distribuição granulométrica, podendo ser obtido um valor médio global.

Por fim, para o cálculo da eficiência de remoção de poluentes de uma determinada bacia de detenção, são necessários os elementos geométricos da mesma, tais como volume, área superficial e profundidade.

O escoamento superficial gerado pela precipitação é caracterizado como uma série de eventos independentes que ocorrem aleatoriamente no tempo. Cada evento em questão é caracterizado pela sua duração $d_{R}$, pelo volume $v_{R}$, o intervalo de tempo desde a precipitação anterior $\delta_{\mathrm{R}}$ (considerando os instantes que definem a metade da duração dos eventos), e o escoamento médio $q_{R}=v_{R} / d_{R}$.

As variáveis $v_{R}, q_{R}, d_{R}$ e $\delta_{R}$ são consideradas aleatórias, independentes entre si e possuem distribuições de probabilidade do tipo Gama, com valores médios iguais a $V_{R}, Q_{R}, D_{R}$ e $\Delta_{R}$, respectivamente, e coeficientes de variação $\mathrm{v}_{\mathrm{v}}, \mathrm{v}_{\mathrm{q}}, \mathrm{v}_{\mathrm{d}}$, e $\mathrm{v}_{\delta}$. Para a aplicação do método assume-se que sejam conhecidas estas estatísticas através da análise de registros de precipitação e escoamento superficial de um período de pelo menos 3 anos.

Em geral, o escoamento superficial não é medido diretamente, assim sendo as estatísticas acima devem ser calculadas a partir da precipitação. Da análise de uma série englobando alguns anos de dados de precipitação com valores medidos em um intervalo de tempo de uma hora ou menos são calculadas as seguintes variáveis: o volume médio de precipitação $\left(\mathrm{V}_{\mathrm{p}}\right)$, a intensidade média $\left(\mathrm{I}_{\mathrm{p}}\right)$, a duração média do evento $\left(\mathrm{D}_{\mathrm{p}}\right)$ e o intervalo médio entre eventos $\left(\Delta_{\mathrm{p}}\right)$. Estes valores médios se referem a eventos individuais, não a valores diários ou mensais.

O volume médio escoado e a vazão média podem ser obtidos pela aplicação do Método Racional. Os valores de duração média e de intervalo médio entre os eventos de escoamento podem ser considerados iguais aos valores calculados para os eventos de precipitação. Os valores dos coeficientes de variação para as quatro estatísticas de escoamento também são considerados iguais aos valores calculados para os eventos de precipitação.

As Equações (7) a (12) descrevem o cálculo das estatísticas de escoamento em função da precipitação.

$\mathrm{Q}_{\mathrm{R}}=\mathrm{I}_{\mathrm{p}} * \mathrm{C}^{*} \mathrm{~A}_{\mathrm{C}}$

onde: $Q_{\mathrm{R}}$ é a vazão média de escoamento $\left(\mathrm{m}^{3} / \mathrm{h}\right)$; $\mathrm{I}_{\mathrm{p}}$ é a intensidade média de precipitação $(\mathrm{m} / \mathrm{h})$;

C é o coeficiente de escoamento da bacia de contribuição;

$\mathrm{A}_{\mathrm{C}}$ é área superficial da bacia de contribuição $\left(\mathrm{m}^{2}\right)$.

$\mathrm{V}_{\mathrm{R}}=\mathrm{V}_{\mathrm{P}} * \mathrm{C}^{*} \mathrm{~A}_{\mathrm{C}}$

onde: $\mathrm{V}_{\mathrm{R}}$ é o volume médio escoado $\left(\mathrm{m}^{3}\right)$;

$\mathrm{V}_{\mathrm{p}}$ é o volume médio de precipitação $(\mathrm{m})$;

C é o coeficiente de escoamento da bacia de contribuição;

$\mathrm{A}_{\mathrm{C}}$ é área superficial da bacia de contribuição $\left(\mathrm{m}^{2}\right)$.

$\Delta_{\mathrm{R}}=\Delta_{\mathrm{p}}$

onde: $\Delta_{\mathrm{R}}$ é intervalo médio entre eventos de escoamento (h);

$\Delta_{\mathrm{p}}$ é intervalo médio entre eventos de precipitação (h).

$\mathrm{v}_{\mathrm{v}}=\mathrm{V}_{\mathrm{Vp}}$

onde: $\mathbf{v}_{\mathrm{v}}$ é coeficiente de variação do volume de escoamento;

$\mathrm{v}_{\mathrm{Vp}}$ é coeficiente de variação do volume de precipitação.

$\mathrm{V}_{\mathrm{q}}=\mathrm{V}_{\mathrm{Ip}}$

onde: $\mathrm{v}_{\mathrm{q}}$ é coeficiente de variação da vazão escoada;

$\mathrm{v}_{\text {Ip }}$ é coeficiente de variação da intensidade de precipitação.

O cálculo da eficiência da remoção dos sólidos em suspensão e dos poluentes associados é feito em duas etapas. Na primeira etapa é feito o cálculo para a condição dinâmica, ou seja, durante os eventos de precipitação. Na segunda etapa é calculada a eficiência estática, durante o período em que não há precipitação, mas existem volumes de água dentro da bacia de detenção. Por fim, calcula-se a eficiência total combinando-se os valores encontrados nas duas situações. 
Para a condição dinâmica, a eficiência da remoção é calculada para cada fração de partículas usando uma equação clássica de sedimentação, equação (13).

$R_{d}=1-\left[1+\frac{1}{n} * \frac{V_{s}}{\frac{Q_{R}}{A_{B}}}\right]^{-n}$

onde: $R_{d}$ é a fração removida da quantidade inicial de sólidos em suspensão;

$\mathrm{V}_{\mathrm{s}}$ é a velocidade média de sedimentação da fração de partículas;

$Q_{R}$ é a vazão média escoada através da bacia de detenção;

$\mathrm{A}_{\mathrm{B}}$ é a área superficial da bacia de detenção;

n é um fator que representa a desempenho da sedimentação.

Esta eficiência é calculada para a vazão na bacia de detenção obtida em função da média $Q_{R}$. Em alguns eventos a vazão de pico através da bacia de detenção poderá ser maior que a média e em outros poderá ser menor. Desta forma a eficiência será maior que a média em alguns casos e menor em outros. Para levar em conta a variação do escoamento ao longo dos eventos na estimativa da eficiência a longo prazo, utiliza-se a Equação (14).

$$
R_{L}=Z *\left[\frac{r}{r-\ln \left(\frac{R_{d}}{Z}\right)}\right]^{(r+1)}
$$

onde: $\quad \mathrm{R}_{\mathrm{L}}$ é a remoção dinâmica a longo prazo;

$\mathrm{R}_{\mathrm{d}}$ a fração removida da quantidade inicial de sólidos em suspensão para a vazão média anual; $\mathrm{r}=1 / \mathrm{v}_{\mathrm{q}}{ }^{2}$;

$\mathrm{Z}$ é a fração máxima removida a taxas de aplicação muito baixas, variando de $80 \%$ a $100 \%$.

Calculada a eficiência para cada fração de partículas, pode-se calcular a eficiência média dinâmica através de uma média aritmética chamada $\mathrm{R}_{\mathrm{LM}}$.

A condição estática de sedimentação ocorre quando não há precipitação, conseqüentemente não há escoamento afluente à bacia de detenção. A eficiência da remoção de sólidos em suspensão neste caso depende diretamente da área da bacia de de- tenção e indiretamente do intervalo de tempo até a próxima condição dinâmica. Isso ocorre porque para frações de sólidos com velocidades de sedimentação muito baixas, o intervalo de tempo entre precipitações pode não ser suficiente para a sedimentação completa. A duração e quando irá ocorrer um período estático são variáveis aleatórias.

$\mathrm{O}$ volume útil da bacia de detenção para a remoção de sólidos não é igual ao volume físico da mesma. Isso ocorre devido à aleatoriedade dos períodos de precipitação, que fazem com que em certos eventos ainda haja volumes de água proveniente de precipitações anteriores. Portanto, para o cálculo da eficiência estática é necessária a determinação do volume efetivo de armazenamento da bacia de detenção.

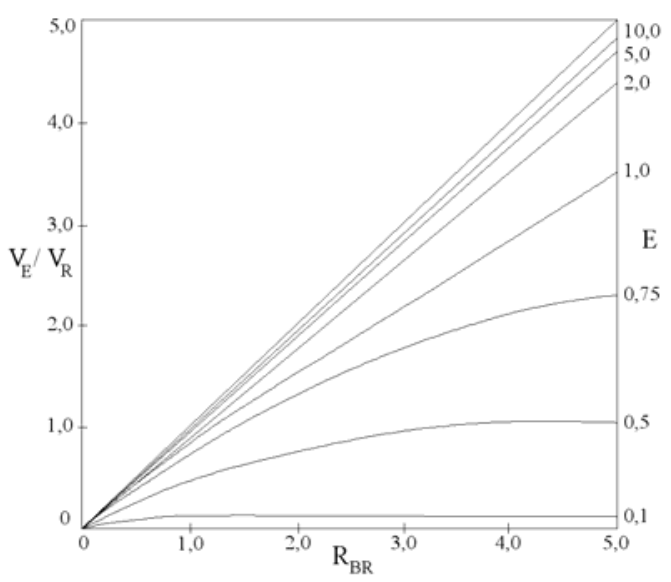

Figura 2 - Efeito de precipitações antecedentes sobre o desempenho de longo prazo sob condições estáticas (adaptado de Driscoll et al., 1986).

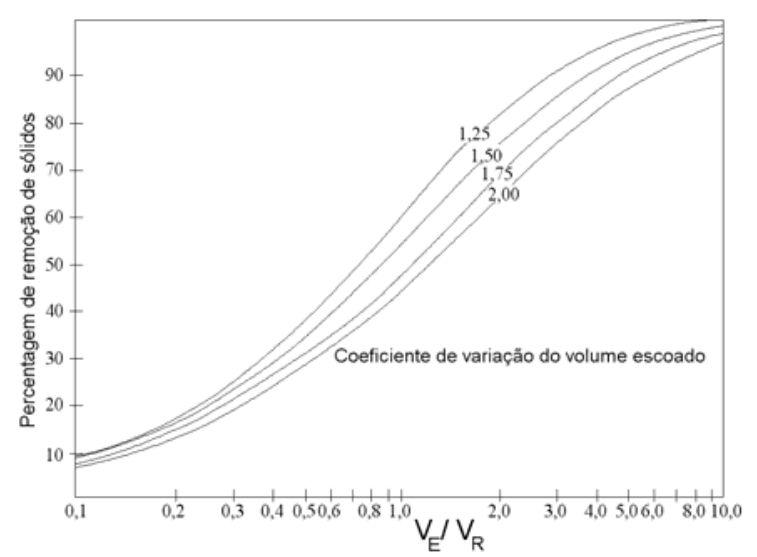

Figura 3 - Desempenho médio de longo prazo de uma bacia de detenção sob condições estáticas (adaptado de Driscoll et al., 1986). 
O cálculo da eficiência da remoção estática não pode ser feito de forma analítica, pois envolve a avaliação de integrais duplas com funções gama. Assim sendo o cálculo deve ser feito através de métodos numéricos. Driscoll et al. (1986) apresenta dois gráficos (Figuras 3 e 4) que permitem o seu cálculo.

Para o uso destas Figuras é necessário o cálculo dos parâmetros $\mathrm{E}$ e $\mathrm{R}_{\mathrm{BR}}$, através das Equações (15) e (16).

$\mathrm{E}=\left(\Delta_{\mathrm{R}} * \mathrm{~V}_{\mathrm{s}} * \mathrm{~A}_{\mathrm{B}}\right) / \mathrm{V}_{\mathrm{R}}$

$\mathrm{R}_{\mathrm{BR}}=\mathrm{V}_{\mathrm{B}} / \mathrm{V}_{\mathrm{R}}$

onde: $\Delta_{\mathrm{R}}$ é intervalo médio entre eventos de escoamento $(\mathrm{h})$;

$\mathrm{V}_{\mathrm{S}}$ é a velocidade de sedimentação da fração de partículas $(\mathrm{m} / \mathrm{h})$;

$A_{B}$ é área superficial da bacia de detenção $\left(\mathrm{m}^{2}\right)$;

$\mathrm{V}_{\mathrm{R}}$ é o volume médio escoado $\left(\mathrm{m}^{3}\right)$;

$\mathrm{V}_{\mathrm{B}}$ é o volume nominal da bacia de detenção em análise $\left(\mathrm{m}^{3}\right)$.

O parâmetro E é uma ponderação entre o intervalo médio de tempo decorrido entre eventos consecutivos e o volume médio escoado. A eficiência da remoção de poluentes nas bacias de detenção aumenta com o aumento deste parâmetro.

$\mathrm{R}_{\mathrm{BR}}$ é a razão entre o volume nominal da bacia de detenção e o volume médio escoado. Quanto maior esta razão, maior será a eficiência da bacia para a remoção de sólidos em suspensão.

Pode-se depreender, através da análise destes dois parâmetros, que para aumentar a eficiência de uma determinada bacia de detenção é necessário o aumento do volume da mesma.

Com os valores calculados com o uso das Equações (15) e (16), utiliza-se a Figura 2 para calcular a relação $V_{E} / V_{R}$. Com esta relação, utiliza-se o valor do coeficiente de variação do volume escoado e o auxilio da Figura 3 para obter o valor da percentagem de remoção dos sólidos na bacia de detenção. Este procedimento é feito para cada fração de sólidos suspensos, com a sua velocidade de sedimentação $V_{S}$.

Calculada a eficiência da remoção estática para cada fração de partículas, pode-se calcular a eficiência média estática através de uma média aritmética, denominando-se esta média de $\mathrm{R}_{\mathrm{DM}}$.

Após terem sido calculadas as eficiências médias, para as condições estáticas e dinâmicas, pode-se calcular a eficiência média combinada para as duas situações através da Equação (17).
$\mathrm{R}_{\mathrm{DLM}}=1-\left(1-\mathrm{R}_{\mathrm{LM}}\right) *\left(1-\mathrm{R}_{\mathrm{DM}}\right)$

Onde: $\mathrm{R}_{\mathrm{DLM}}$ é a eficiência média combinada;

$\mathrm{R}_{\mathrm{LM}}$ é a eficiência média dinâmica;

$\mathrm{R}_{\mathrm{DM}}$ é a eficiência média estática.

\section{RESULTADOS}

Para a execução do estudo, foram utilizados parte dos dados de precipitação compilados e organizados por Bemfica (2000). A série usada inicia em setembro de 1974 e termina em abril de 1998. Os dados relativos à precipitação são a data de ocorrência, o horário com resolução de cinco minutos e volume com resolução de $0,1 \mathrm{~mm}$. Para a execução da análise foi necessário agrupar os dados em um único arquivo de planilha eletrônica, pois uma série de cálculos automatizados através de programação de macros em Visual Basic foram feitos com a base nos dados. Assim sendo os dados foram importados e organizados em uma única seqüência, contendo todos os eventos consecutivamente. Os dados brutos para o período totalizam 196.985 linhas, ou intervalos de tempo com precipitação.

Foi adotado um intervalo de tempo de 12 horas sem precipitação para que se configurasse a ocorrência de um novo evento. Utilizando-se este critério, foram individualizados um total de 1.932 eventos a partir dos dados brutos. Para cada evento foi calculado o total precipitado, a duração, a intensidade média e um intervalo de tempo desde o evento anterior considerando os instantes que definem a metade da duração dos eventos. Os valores médios e desvios padrões para estas estatísticas estão na Tabela 1 .

Tabela 1 - Estatísticas da precipitação no posto do Oitavo Distrito do INMET.

\begin{tabular}{lcc}
\cline { 2 - 3 } & Média & $\begin{array}{c}\text { Desvio } \\
\text { Padrão }\end{array}$ \\
\hline Precipitação $(\mathrm{mm})$ & 15,4 & 19,0 \\
\hline Duração $(\mathrm{h})$ & 10,7 & 12,4 \\
\hline Intensidade $(\mathrm{mm} / \mathrm{h})$ & 2,2 & 2,8 \\
\hline Intervalo $(\mathrm{h})$ & 107,3 & 93,4 \\
\hline
\end{tabular}

Para a determinação do volume maximizado, alguns eventos daqueles 1.932 que foram individualizados foram eliminados do processo. Em primeiro lugar, é preciso definir qual é o volume esti- 
mado para as perdas iniciais. A literatura cita valores de 1,45 a $2,9 \mathrm{~mm}$ e em alguns trabalhos, tal como Urbonas (1996) foi adotado o valor de 2,54 mm (0,1 polegada). O valor adotado, portanto, pode ser considerado um tanto arbitrário. Porém, manteve-se o valor de 2,54 $\mathrm{mm}$ para permitir comparações com os resultados obtidos em outros trabalhos.

Também foi necessário eliminar os valores do extremo superior, em termos de volume, para evitar que estes poucos valores tenham um peso significativo sobre os resultados. No trabalho Urbonas (1996), foi utilizado o percentil 99,5. Da mesma forma que para as perdas iniciais, a escolha é um tanto arbitrária. Foi adotado o valor do percentil 99,5 .

Da aplicação do primeiro critério restaram 1.457 eventos, e após a aplicação do segundo critério, restaram 1.444 dos 1.932 eventos iniciais. O volume do evento que corresponde ao percentil 99,5 $\left(\mathrm{P}_{\mathrm{M}}\right)$ é de $96,95 \mathrm{~mm}$, ocorrido no dia 14/11/1993. O tempo de esvaziamento considerado no algoritmo foi de 24 horas.

Com estes eventos foi determinado o volume maximizado, para coeficientes de escoamento de 0,1 a 1 com variação de 0,1 . Para cada valor de coeficiente de escoamento o volume da bacia de detenção $\left(\mathrm{P}_{\mathrm{P}}\right)$ foi variado, iniciando em $0 \mathrm{~mm}$, recebendo incrementos de $0,33 \%$ até atingir cerca de $29 \%$ do valor do maior evento, que corresponde a 28,6 mm. As simulações mostraram que volumes de detenção acima de $29 \%$ sempre capturam todo o volume escoado.

Tabela 2 - Volume maximizado em relação ao coeficiente de escoamento.

\begin{tabular}{cc}
\hline $\begin{array}{c}\text { Coeficiente de } \\
\text { escoamento }\end{array}$ & $\begin{array}{c}\text { Volume maximizado } \\
(\mathrm{mm})\end{array}$ \\
\hline 0,0 & 0,0 \\
\hline 0,1 & 3,6 \\
\hline 0,2 & 6,5 \\
\hline 0,3 & 9,6 \\
\hline 0,4 & 11,3 \\
\hline 0,5 & 13,7 \\
\hline 0,6 & 15,7 \\
\hline 0,7 & 17,1 \\
\hline 0,8 & 19,2 \\
\hline 0,9 & 20,3 \\
\hline 1,0 & 22,5 \\
\hline
\end{tabular}

Após isto, foi calculada a declividade da reta tangente a cada um dos pontos definidos pela razão de captura e procurados aqueles mais próximos ao valor de 1:1 para encontrar o ponto de maximização. Em seguida foram calculados, através de interpolações lineares, os valores exatos da razão de captura e do volume da bacia de detenção que definem a declividade unitária da reta tangente.

Na Tabela 2 estão os volumes maximizados em $\mathrm{mm}$ para cada coeficiente de escoamento. Estes valores significam que, para uma bacia de contribuição com determinado coeficiente de escoamento, é necessária uma bacia de detenção com um volume igual ao produto entre a área de bacia de contribuição e o volume maximizado correspondente, respeitadas as unidades de medida.

Para facilitar o uso dos valores contidos na Tabela 2, foram ajustadas duas curvas aos mesmos, resultando em uma reta e uma equação do terceiro grau com coeficientes $\mathrm{R}^{2}$ de Nash de 0,96 e 0,99, respectivamente Equações (18) e (19). Na Figura 4 é mostrado um gráfico contendo os pontos calculados e a curvas ajustadas. Pode-se observar que a equação de terceiro grau ajusta-se melhor aos volumes calculados. O ajuste através de uma reta subestima os volumes até o coeficiente de escoamento de 0,7 e superestima os demais volumes, porém esta equação pode ser usada em situações onde não é necessária uma grande precisão.

$\mathrm{V}=24,3 * \mathrm{C}, \mathrm{R}^{2}=0,96$

$\mathrm{V}=11,6^{*} \mathrm{C}^{3}-27,4 * \mathrm{C}^{2}+38,1 * \mathrm{C}, \mathrm{R}^{2}=0,99$

Onde: V é o volume maximizado ( $\mathrm{mm})$; C é o coeficiente de escoamento.

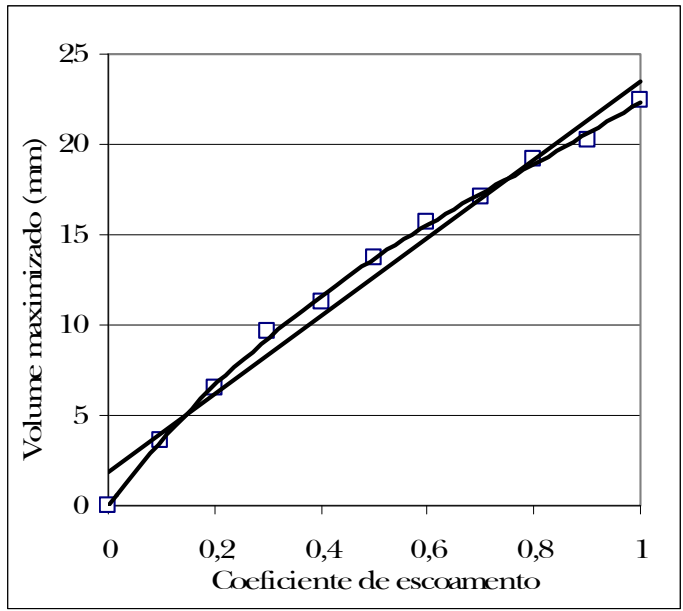

Figura 4 - Ajustamento de equações de primeiro e terceiros graus aos volumes maximizados (adaptado de SILVA, 2009). 
Urbonas e Starhe (1993) realizaram uma análise da sensibilidade da eficiência da remoção de poluentes em relação ao volume anual capturado. Nesta análise foram calculadas as eficiências da remoção de poluentes para volumes inferiores e superiores ao volume maximizado. Para o volume maximizado a eficiência foi de $88 \%$, para um volume equivalente a $70 \%$ do maximizado a redução na eficiência foi de apenas $2 \%$ e para atingir uma eficiência de $90 \%$ foi necessário aumentar o volume capturado em mais de $200 \%$. Esta análise mostrou de que uma redução de volume em relação ao volume maximizado não reduz significativamente a eficiência na remoção de poluentes.

Por esta razão é que alguns estados americanos adotam percentagens do volume anual ao invés do volume maximizado como parâmetro de projeto para controle da poluição difusa. Pode-se citar o estado da Geórgia (Amec, 2001) que adota um volume igual a $85 \%$ do volume anual, enquanto que em Denver (Udfcd, 2002) é adotado o valor de $80 \%$ e em Maryland utiliza-se $90 \%$.

Assim sendo, decidiu-se calcular as volumes correspondentes a 80,85 e $90 \%$ de captura e avaliar a eficiência dos mesmos utilizando-se a metodologia descrita por Driscoll et al. (1986). O cálculo do volume capturado foi feito com uma metodologia semelhante àquela adotada para o cálculo do volume maximizado, com exceção de que o volume procurado é aquele correspondente à percentagem de captura desejada e não ao volume maximizado. As Equações (20), (21) e (22) correspondem aos volumes encontrados em função do coeficiente de escoamento.

$$
\begin{aligned}
& \mathrm{V}_{80}=14,7^{*} \mathrm{C} \\
& \mathrm{V}_{85}=16,8^{*} \mathrm{C} \\
& \mathrm{V}_{90}=19,5^{*} \mathrm{C}
\end{aligned}
$$

Uma vez determinados os volumes de controle para o caso maximizado e para os casos em que se deseja a captura de 80,85 e $90 \%$ do volume anual, o passo seguinte foi a verificação da eficiência da remoção dos poluentes através da sedimentação para cada um destes casos. Os resultados em percentagem média de remoção de sólidos, para bacias de detenção profundidade de 1 metro estão na Tabela 3. Como já era esperado, a eficiência aumenta a medida que aumenta a percentagem de captura.
Tabela 3 - Eficiência média na remoção de poluentes

\begin{tabular}{cc}
\hline Captura $(\%)$ & Eficiência $(\%)$ \\
\hline 80 & 79,42 \\
\hline 85 & 82,26 \\
\hline 90 & 84,75 \\
\hline Maximizado & 91,72 \\
\hline
\end{tabular}

Para a determinação dos custos, foi adotada a mesma bacia de amortecimento fictícia usada para o cálculo da eficiência da remoção de sólidos. A bacia tem seu volume obtido através da escavação do solo, sendo o fundo e os taludes cobertos com grama. Ela possui formato retangular, com o lado maior igual a duas vezes o lado menor, e possui taludes com inclinação de 1:2. Os serviços necessários para sua execução são típicos de obras de drenagem executadas em Porto Alegre, sendo os seguintes: escavação mecânica, escavação manual, transporte do material escavado até $2 \mathrm{~km}$, transporte por $\mathrm{km}$ excedente, plantio de grama de campo, execução de cerca e dispositivos de entrada e saída. Os preços dos serviços foram obtidos da tabelas oficiais da Prefeitura Municipal de Porto Alegre, com data base de agosto de 2008 (Porto Alegre, 2009).

Foram calculados os custos por metro cúbico para o caso maximizado e para os casos em que se deseja a captura de 80,85 e $90 \%$ do volume anual. Os resultados médios em $\mathrm{R} \$ / \mathrm{m}^{3}$ estão apresentados na Tabela 4. Pode-se observar que os custos unitários tendem a diminuir com o aumento da percentagem de captura e, conseqüentemente, do volume a ser armazenado. Em parte isto se deve à diluição de custos, pois os serviços de execução de cerca e dispositivos de entrada e saída, que têm um valor unitário caro em relação aos outros, tendem a ter menor peso à medida que o volume aumenta.

Tabela 4 - Custo unitário das bacias

\begin{tabular}{cc}
\hline Captura $(\%)$ & Custo $\left(\mathrm{R} \$ / \mathrm{m}^{3}\right)$ \\
\hline 80 & 96,28 \\
\hline 85 & 92,09 \\
\hline 90 & 87,91 \\
\hline Maximizado & 77,18 \\
\hline
\end{tabular}

Os custos por $\mathrm{m}^{3}$ de bacia de detenção não podem ser simplesmente comparados entre si, pois as percentagens de captura e, conseqüentemente as eficiências de remoção dos poluentes são diferentes entre elas. Procurou-se criar então um índice que 
pudesse ser usado para comparar as diferentes eficiências apresentadas pelas bacias e seus respectivos custos, sendo algo análoga ao benefício versus custo da economia. Este índice foi calculado dividindo-se a eficiência da remoção de poluentes, que pode ser considerado um benefício, pelo se respectivo custo por $\mathrm{m}^{3}$. Os resultados em percentagem de remoção, divididos por $\mathrm{R} \$ / \mathrm{m}^{3}$, são apresentados na Tabela 5 .

Tabela 5 - Relação eficiência/custo das bacias

\begin{tabular}{cc}
\hline Captura $(\%)$ & Índice $(\% / \mathrm{R} \$)$ \\
\hline 80 & 0,76 \\
\hline 85 & 0,84 \\
\hline 90 & 0,94 \\
\hline Maximizado & 1,24 \\
\hline
\end{tabular}

Através destes resultados, verifica-se que os índices aumentam à medida que se aumenta a percentagem de captura. Isto significa que, dentro dos critérios adotados neste estudo, a solução ideal é adotar o volume maximizado para o controle da poluição difusa, pois tem a melhor eficiência de remoção de poluentes e o melhor índice de benefício. Além disto, existem os benefícios ambientais adicionais de se lançar menos carga poluente nos corpos hídricos, que são difíceis de avaliar e não estão computados neste estudo.

\section{COMPARAÇÃO COM CRITÉRIOS UTILIZADOS EM OUTROS LOCAIS}

Procurou-se encontrar quais os critérios utilizados para o controle da poluição difusa em locais no exterior, que tivessem características semelhantes, em relação à precipitação, com Porto Alegre.

Tabela 5 - Locais e volumes necessários de amortecimento

\begin{tabular}{cc}
\hline Local & Volume $\left(\mathrm{m}^{3} / \mathrm{ha}\right)$ \\
\hline Virginia & 127,00 \\
\hline Carolina do Norte & 127,00 \\
\hline Knoxville & 146,05 \\
\hline Porto Alegre & 137,46 \\
\hline
\end{tabular}

Para fins de comparação com Porto Alegre, foram calculados os volumes necessários de captura usando os critérios de cada um dos locais. Adotou-se um coeficiente de escoamento de 0,5 e uma área de contribuição de 1 hectare. Na Tabela 5 estão os volumes encontrados.

O valor encontrado para Porto Alegre ficou dentro do intervalo de valores dos três locais selecionados.

\section{CONCLUSÃO}

Através da combinação da metodologia de maximização de captura de volume de escoamento superficial e da metodologia para verificação da eficiência da remoção de sólidos em suspensão, foi obtido um modelo para pré-dimensionamento de bacias de detenção para controle da poluição difusa. Este modelo além de ser otimizado em termos de volume capturado é o que, dentre as alternativas analisadas, apresenta a melhor relação beneficio/custo, avaliado em termos da eficiência de remoção de poluentes e custo de implantação da estrutura. As simulações mostraram que o volume de detenção necessário para o controle da poluição difusa é de $157 \mathrm{~m} 3 /$ ha para um coeficiente de escoamento de 0,6 , variando de 36 a $225 \mathrm{~m} 3 /$ ha para coeficientes de escoamento de 0,1 e 1,0 , respectivamente.

O modelo proposto é facilmente integrável à metodologia de dimensionamento de bacias de detenção para controle de cheias em uso no Município de Porto Alegre, bastando para isso que sejam somados os volumes calculados para o controle das cheias e da poluição difusa, e que seja feito o dimensionamento das estruturas de saída de modo que o volume de controle qualitativo seja drenado em um tempo igual ou superior a 24 horas. Considerando o critério atual de dimensionamento para controle de cheias, a adoção do controle qualitativo implicaria em um aumento médio de $55 \%$ no volume das bacias de detenção.

\section{REFERÊNCIAS}

AMEC. (2001). Georgia Stormwater Management Manual. Volume 2: Technical Handbook. Atlanta Regional Commission.

BEMFICA, D. C. 2000. Verificação da Aplicabilidade de Padrões de Chuva de Projeto a Porto Alegre - RS Brasil. Porto Alegre: UFRGS- Curso de Pós- 
Graduação em Recursos Hídricos e Saneamento Ambiental. 130f. Dissertação (Mestrado).

CASQA (2003). Stormwater Best Management Practice Handbook. New Development and Redevelopment. California Stormwater Quality Association, Los Angeles, California.

CHANEY, R. H. (1982). Stormwater Detention Facilities in Moddletown, Ohio. 1982 International Symposium on Urban Hydrology, Hydraulics and Sediment Control. University of Kentucky, Lexington, Kentuky. pp. 367377.

CLAR, M. L. e BARFIELD, B. J. (2004). Stormwater Best Management Practice Design Guide. Report No. EPA /600/R-04/121 U.S. EPA, Washington, DC.

DRISCOLL, E. D., et al, (1986). Methodology for Analysis of Detention Basins for Control of Urban Runoff Quality. Report No. EPA 440/5-87-01 (NTIS No. PB87116562), U.S. EPA, Washington, DC.

GUO, J. C. Y. E URBONAS, B. (1996). Maximized Detention Volume Determined by Runoff Capture Ratio. Journal of Water Resources and Planning. January/February 1996, pp 33-39

MARYLAND. (2000). Maryland Stormwater Design Manual. Vol I e II. Maryland Department of the Environment. Baltimore, Maryland.

PORTO ALEGRE. Prefeitura Municipal de Porto Alegre. (2009). Secretaria Municipal de Obras e Viação. Tabela de preços para obras viárias. Disponível em: http://lproweb.procempa.com.br/pmpa/prefpoa/smov/ usu_doc/tp_ago20082.pdf. Acessado em 17/09/09.

PORTO, M. F. A. (1995). Aspectos Qualitativos do Escoamento Superficial em Áreas Urbanas. In: TUCCl, C. E. M., PORTO R. L. L., BARROS, M. T. (Org). Drenagem Urbana. Porto Alegre: Editora da Universidade UFRGS:ABRH. v.5. Porto Alegre, pp 387-428.

SILVA, M. K. 2009. Modelo para Pré-dimensionamento de Bacias de Detenção para Controle da Poluição Difusa das Águas Pluviais no Município de Porto Alegre. Porto Alegre: UFRGS- Curso de Pós-Graduação em Recursos Hídricos e Saneamento Ambiental. 140f. Dissertação (Mestrado).

UDFCD, 2002. Drainage Criteria Manual. Volume 3. Urban Drainage and Flood Control District, Denver, Colorado.

URBONAS, B. e STAHRE, P. (1993). Stormwater Best Management Practices and Detention. Prentice Hall, Englewood Cliffs, New Jersey. 450 p.

\section{Model For Pre-sizing Detention Ponds to Control Diffuse Pollution of Stormwater in Porto Alegre}

\begin{abstract}
Diffuse pollution of stormwater is one of several environmental impacts caused by urbanization. Detention ponds can control peak flows, but can also be used to control stormwater quality through the natural sedimentation of pollutants. A model was developed to pre-size detention ponds for stormwater quality control in Porto Alegre, It was based on a methodology to maximize capture volume and a methodology for calculating the efficiency of removal pollutants. Rainfall series from Porto Alegre were used to develop the model. Simulations showed that detention volumes needed for quality control are $157 \mathrm{~m}^{3} / \mathrm{h}$ a for a runoff coefficient of 0.6, typical of urban areas, ranging from 36 to $225 \mathrm{~m}^{3} /$ ha for runoff coefficients from 0.1 to 1.0, respectively. The proposed model was compared with models used in the United States with similar rainfall characteristics, resulting in compatible detention volumes.
\end{abstract}

Key-words: detention pond, diffuse pollution, stormwater. 